\title{
Flora do Mato Grosso do Sul: Myrtaceae
}

\section{Carolyn Elinore Barnes Proença ${ }^{1}$, Lucia Helena Soares-Silva ${ }^{1}$, Daniel Villarroel ${ }^{1,2}$, Kadja Milena Gomes-Bezerra', Priscila Oliveira Rosa ${ }^{3}$, Jair Eustáquio Quintino de Faria' \& Marcos Sobral ${ }^{4}$}

\author{
1. Universidade de Brasília, Programa de Pós-Graduação em Botânica, Depto. de Botânica, Campus Darcy Ribeiro, \\ CEP 70.919-900, Brasília, DF, Brasil. cproenca@unb.br \\ 2. Museo de Historia Natural Noel Kempff Mercado, Depto. de Botánica, \\ Av. Irala 565, Casilla 2489. Santa Cruz, Bolivia. \\ 3. Jardim Botânico de Brasília, Diretoria de Fitologia, Área Especial SMDB Estação Ecológica Jardim Botânico de Brasília, \\ Setor de Mansões Dom Bosco (Lago Sul), CEP 71680-001, Brasília, DF, Brasil. \\ 4. Universidade Federal de São João del Rei, Departamento de Ciências Naturais, Campus Dom Bosco - CDB, \\ Praça Dom Helvécio, 74 - Fábricas, CEP 36301-160 - São João del Rei, MG, Brasil.
}

Recebido em 27.IX. 2014

Aceito em 15.VII.2016

DOI 10.21826/2446-8231201873s277

RESUMO - O conhecimento da família Myrtaceae no Mato Grosso do Sul foi tardio em relação às outras unidades da federação. Na Flora Brasiliensis foram citadas nove espécies para o que é hoje território do estado. Myrtaceae apresenta atualmente registro de 81 espécies no Mato Grosso do Sul. A importância da família é variável em estudos florísticos, sendo em geral maior no Cerrado e menor no Pantanal, podendo se apresentar até como a segunda família mais diversa. Ocorrem dez gêneros: Blepharocalyx O.Berg (uma espécie), Calyptranthes Sw. (duas espécies), Campomanesia Ruiz \& Pav. (seis espécies), Eugenia L. (35 espécies), Myrcia DC. (21 espécies), Myrcianthes O.Berg (uma espécie), Myrciaria O.Berg (quatro espécies), Pimenta Lindl. (uma espécie), Plinia L. (duas espécies) e Psidium L. (nove espécies). O presente estudo resultou em um incremento de 47 espécies e saída de sete espécies em relação à última Lista de Espécies da Flora do Brasil, resultando em um ganho líquido de 40 espécies em relação a esta listagem.

Palavras-chave: Cerrado, Eugenia, Myrcia, Pantanal, Psidium

\begin{abstract}
Flora of Mato Grosso do Sul: Myrtaceae. Studies on the family Myrtaceae started later in Mato Grosso do Sul than in other Brazilian states. In Flora Brasiliensis, nine species were cited for what is now the state's territory. There are now 81 species of Myrtaceae recognized for Mato Grosso do Sul. The importance of the family in floristic studies in the state is variable, and is generally higher in Cerrado vegetation than in the Pantanal, and in some studies it was found to be the second most species-rich family. Ten genera occur: Blepharocalyx O.Berg (one species), Calyptranthes Sw. (two species), Campomanesia Ruiz \& Pav. (six species), Eugenia L. (35 species), Myrcia DC. (21 species), Myrcianthes O.Berg (one species), Myrciaria O.Berg (four species), Pimenta Lindl. (one species), Plinia L. (two species) and Psidium L. (nine species). This study resulted in an addition of 47 species and the elimination of seven species in relation to the Species List of the Brazilian Flora, resulting in a net gain of 40 species.
\end{abstract}

Keywords: Eugenia, Myrcia, Pantanal, Psidium, Savanna

\section{INTRODUÇÃO}

O conhecimento da família Myrtaceae em Mato Grosso do Sul foi tardio em relação às outras unidades da federação. $\mathrm{Na}$ Flora Brasiliensis (Berg 1857, 1859), foram citadas nove espécies (10 coletas) de Myrtaceae para o estado, todas feitas por L. Riedel na famosa expedição organizada pelo Barão von Langsdorff do Rio de Janeiro a Cuiabá. Entre agosto e novembro de 1826, a expedição cortou o atual estado do Mato Grosso do Sul quando deixou o Rio Paraná e adentrou o Rio Pardo pela sua foz, navegando em direção a Camapuã (Silva 1997). Dentre as nove espécies originalmente descritas, três permanecem aceitas e as demais foram sinonimizadas. Dentre essas três espécies ainda aceitas, duas são tipificados por material coletado no Mato Grosso do Sul: Eugenia angustissima O.Berg e E. langsdorffii O.Berg. Barbosa
Rodrigues (1903) descreveu uma espécie de Psidium L. entre as suas "Myrtacée du Paraguay" para Bela Vista, hoje parte do estado, mas esta é um sinônimo de Psidium guajava L. (Govaerts et al. 2012). Em 2009 foi descrita Eugenia matogrossensis Sobral (Sobral 2008) baseado em material do MS sendo que outra espécie descrita em 2012 para o Mato Grosso (Eugenia neomattogrossensis Mazine) também ocorre em MS (Mazine 2012).

A Lista de Espécies Flora do Brasil registrou 41 espécies de Myrtaceae ocorrentes em Mato Grosso do Sul (Sobral et al. 2012), uma baixa diversidade se comparada com estados vizinhos como Paraná (227 espécies), São Paulo (264 espécies) e Mato Grosso (81 espécies). Até então, a única compilação disponível era a obra de Dubs (1998) que registrava 102 espécies para Mato Grosso e Mato Grosso do Sul conjuntamente. 
Myrtaceae apresenta importância variável em estudos florísticos no estado, sendo em geral menor no Pantanal e maior no Cerrado (Ratter et al. 1988). Levantamentos florísticos no Complexo Aporé-Sucuriú na divisa Mato Grosso do Sul/Goiás, com levantamentos em seis sítios em Mato Grosso do Sul e dois em Goiás (Pott et al. 2006) registraram um total de 1.579 espécies de angiospermas. Myrtaceae ficou na oitava posição geral em número de espécies, e entre as cinco famílias mais diversas no sítio mais rico entre os inventariados (Sítio 4). Este mesmo estudo salientou que quatro famílias apresentaram "grandes dificuldades taxonômicas e necessitando de coletas e estudos mais intensos" entre as quais está Myrtaceae, já que dos 50 táxons registrados, 17 permaneceram determinados apenas a nível de gênero e quatro a nível de família.

Romagnolo \& Souza (2000), em análise florística e estrutural de florestas ripárias no município de Taquarussu - MS, evidenciaram Myrtaceae como a família com maior riqueza de espécies da pesquisa, onde Eugenia L. e Psidium L. também figuraram entre os gêneros mais ricos. Em estudo sobre alguns gêneros de Myrtaceae no Rio Paraná, estados do Paraná e Mato Grosso do Sul, as autoras destacaram a importância da família para os ambientes ripários brasileiros (Romagnolo \& Souza 2004) o que já havia sido comentado por Langsdorff em 1826 (Silva 1997).

Bueno et al. (2005) salientam que algumas Myrtaceae também são utilizadas como plantas medicinais por tribos indígenas do Mato Grosso do Sul. A população indígena Kaiowá/Guarani, que vem enfrentando a perda de suas terras tradicionais, faz uso de três espécies da família (Eugenia uniflora L., Myrcianthes pungens (O.Berg) D.Legrand, e Psidium guajava L.) com a finalidade de produzir medicamentos. Myrtaceae é a terceira família botânica em número de espécies mais utilizada por essa etnia indígena.

Quando se consideram as árvores, Myrtaceae também apresenta uma elevada riqueza de espécies. No Maciço do Urucum, Silva et al. (2000) registraram Myrtaceae como a segunda família com maior número de espécies arbóreas (13 espécies), sendo que a primeira foi Fabaceae (56 espécies). Resultado similar foi obtido para um estudo em florestas estacionais ribeirinhas, duas em Bonito - MS e três no Parque Estadual da Serra da Bodoquena (Baptista-Maria et al. 2009), onde Myrtaceae, com 21 espécies, manteve a segunda posição em riqueza de espécies, logo após as Fabaceae (51 espécies).

No entanto, em uma mata ciliar da cidade de Miranda no Pantanal, Myrtaceae foi inexpressiva, sendo representada por apenas 10 dos 318 indivíduos amostrados, e pertencentes a duas espécies: Eugenia egensis DC. e Psidium kennedyanum Morong, em um total de 39 espécies (Bueno et al. 2011). É talvez digno de nota que as duas famílias mais ricas em espécies (Arecaceae e Fabaceae) nesta mata apresentavam três espécies cada. Em uma floresta estacional semidecídua (perturbada), na mesma localidade, apenas quatro dos 320 indivíduos eram Myrtaceae, pertencentes a duas espécies, entre as 46 espécies registradas (Battilani et al. 2011), sendo que a mais frequente foi a pioneira Psidium guajava L. (goiabeira).

\section{Principais Grupos de Pesquisa}

A família Myrtaceae atualmente conta com vários estudiosos no país. Os mais relevantes para o Mato Grosso do Sul são os grupos do Centro-Oeste e do Sudeste. No grupo do Sudeste elencamos Marcos Sobral (UFSJ), o especialista mais ativo do Brasil, e um núcleo iniciado pela Dra. Graziela Barroso que teve a participação de Ariane Luna Peixoto e Marli Pires de Lima, sendo Marcelo da Costa Souza seu expoente atual (Plinia L. e Neomitranthes Kausel ex D.Legrand). Outros mirtólogos do Sudeste são Fiorella Mazine Capelo (Eugenia L.), Matheus Fortes Santos (Myrcia DC.), Duane Lima (Myrcia DC.), Mariana Bünger (Eugenia L.) e em treinamento o pós-graduando Augusto Giaretta (Calycolpus O. Berg).

O grupo do Centro-Oeste, liderado pelas profs. Carolyn Proença e Lucia Helena Soares-Silva, tem sido bastante expressivo na formação de jovens mirtólogos, podendo ser citados (alfabeticamente): Daniel Villarroel Segarra (mestrado e doutorado), Jair Eustáquio Quintino de Faria (mestrado e doutorado), Kadja Milena Gomes Bezerra (doutorado), Plauto Simão de Carvalho (mestrado e doutorado) e Priscila Oliveira Rosa (doutorado), a maioria co-autores deste trabalho.

Há também vários mirtólogos nas regiões Norte e Nordeste do Brasil, mas, excetuando Marla Ibrahim (Campomanesia Ruiz \& Pav., co-autora de Myrtaceae de Goiás e Tocantins) não há determinações dos mesmos no material do estado. Os estudos de Lucas et al. (2011) foram importantes na circumscrição de Myrcia adotada no presente trabalho.

\section{Principais Lacunas de Conhecimento}

A maior lacuna é a falta de um tratamento taxonômico completo de Myrtaceae para o estado de Mato Grosso do Sul. O estado abriga uma biota singular, e mesmo havendo estudos apontando a reduzida importância de Myrtaceae na flora do Pantanal, apenas com um tratamento taxonômico será possível apontar a real diversidade e importância da família no estado. A situação é agravada pelo avanço das atividades agropecuárias que estão alterando o ambiente antes que a flora possa ser conhecida.

Mesmo para as espécies conhecidas e já catalogadas para o estado, é necessário um estudo aprofundado que demonstre como as características ambientais singulares dessa área incidem na variabilidade morfológica da vegetação. Há diversas coletas que não foram identificadas, podendo ser indivíduos anômalos ou atípicos de espécies já descritas ou espécies novas.

\section{Principais Acervos e Perspectivas de Pesquisa para o Grupo nos próximos 10 anos}

Os herbários com representação mais expressiva das Myrtaceae de MS, disponíveis on line, são: CGMS (882 ou $31,35 \%$ dos espécimes coletados), CPAP (271), HUEM 
(257), UB (247), MBM (244), SP (193), RB (182) e outros (537 ou 19,1\% do total) segundo o SpeciesLink (2012). Há muitas coletas recentes no CGMS e no RB, respectivamente de G. Damasceno Júnior e G. Martinelli.

Com a finalização da Flora de Goiás e Tocantins esperase uma colaboração entre os pesquisadores do Centro-Oeste para que, na impossibilidade de se tratar esse material no MS, ele seja enviado via empréstimo ou doação para que esses projetos possam ser concretizados.

\section{MATERIAL E MÉTODOS}

Inventários da flora do estado foram consultados (Ratter et al. 1988, Dubs 1998, Silva et al. 2000, Pott et al. 2006, Battilani et al. 2011, Bueno et al. 2011), e os herbários CEN, CGMS, K e UB foram visitados pessoalmente (acrônimos segundo Thiers 2012).

Os sites SpeciesLink (2012), Lista de Espécies Flora do Brasil (Sobral et al. 2012) e FloResCer (2012) também foram consultados. Para compor o checklist, coletas que preferencialmente preenchiam os dois seguintes quesitos foram selecionadas: 1) sua identidade havia sido checada por um dos autores; e 2) estavam presentes em um dos herbários do estado.

Quando um destes quesitos não pode ser preenchido foram citadas duas coletas, sendo referido primeiro o material confirmado pelos autores, e a seguir uma coleta depositada em um dos herbários do estado e identificada por um especialista da família, listados alfabeticamente a seguir: Bruce Holst, Fiorella Mazine, Leslie Landrum, Marcelo de Souza, Maria Lucia Kawasaki, Marla Ibrahim e Rogers McVaugh. Quando vários materiais atendiam a estas condições eram selecionados preferencialmente os que estavam presentes no maior número de herbários. Em casos em que nenhuma coleta preenchia ambas exigências, foi listada uma coleta cuja identidade não pode ser checada, mas que foi identificada por um especialista da família.

Os autores que confirmaram a determinação estão citados após a coleta por meio das suas iniciais seguidos de $!$ em sobrescrito: $\mathrm{CEBP}=$ Carolyn E.B. Proença; $\mathrm{DVS}=$ Daniel Villarroel Segarra; JEQF = Jair E.Q. Faria; KMGB = Kadja M. Gomes-Bezerra; LHSS = Lucia H.Soares-Silva; MS = Marcos Sobral; POR = Priscila Oliveira Rosa.

\section{RESULTADOS E DISCUSSÃO}

Houve um incremento recente de 47 espécies e a exclusão de sete espécies em relação à Lista de Espécies Flora do Brasil (que elencava 40 espécies em 2012), resultando em um ganho líquido de 40 espécies em relação a essa lista (Sobral et al. 2012). Há ainda espécies cuja ocorrência é bastante provável, mas não pode ser inequivocamente comprovada: Myrcia fenzliana O.Berg foi citada para Goiás por Sobral et al. (2012), mas ocorre também na Bolívia (Mamani et al. 2011), e há uma coleta identificada por não-especialista; Campomanesia velutina O.Berg, com ocorrência em Goiás e no Paraná (Sobral et al.
2012), também é de ocorrência provável com identificações on line não comprovadas por especialista. O registro atual então é de 81 espécies (Quadro 1), mas estima-se que poderá aumentar no futuro. Em termos de gêneros, o número aumentou de sete para dez desde $2012 \mathrm{com}$ a inclusão dos gêneros Calyptranthes Sw., Myrcianthes O.Berg e Pimenta Lindl. em relação à Lista de Espécies Flora do Brasil (Sobral et al. 2012).

As espécies citadas por Sobral et al. (2012), mas não confirmadas e que deverão ser excluídas da flora do estado são: Campomanesia rufa (O.Berg) Nied., Eugenia herbacea O.Berg, Eugenia livida O.Berg, e Psidium riparium Mart. ex DC., sendo o principal motivo da exclusão a circunscrição adotada além de duas sinonimizações feitas no presente trabalho. Também foi excluída Psidium guajava L. por não ser nativa, embora ocorra como subespontânea (Battilani et al. 2011).

\section{Notas taxonômicas}

O presente estudo propõe dois novos sinônimos por motivos que serão apresentados a seguir:

Eugenia angustissima O.Berg., Fl. Bras. 14(1) Suppl.: 569. 1859. Eugenia herbacea O.Berg, Fl. Bras. 14(1) Suppl.: 570. 1859. Nov. Syn. Tipo: Brasil. Mato Grosso do Sul. In campis arenosis ad Rio Pardo. Riedel s.n. (LE)

Eugenia angustissima O.Berg e E. herbacea O.Berg foram descritas no Flora Brasiliensis por Berg (1859), fundamentadas em coletas feitas em campos arenosos ao longo do Rio Pardo, MS, por Riedel na expedição de Langsdorff, respectivamente, em setembro e outubro de 1826. As folhas do tipo de E. angustissima são mais estreitas que as do tipo de $E$. herbacea mas a forma do botão floral e os longos pedicelos são extremamente semelhantes. Esta espécie hoje tem sua variabilidade intra-específica bem conhecida já que é amplamente distribuída na região dos cerrados (Proença 1994, Faria 2010, Mamani et al. 2011) e não se justifica a manutenção de duas espécies distintas baseadas em diferenças da puberulência dos ramos, e pedicelos unifloros em E. angustissima versus bifloros em $E$. herbacea, já que aparecem os dois tipos, às vezes no mesmo ramo. Os nomes têm igual prioridade, mas o nome $E$. angustissima é preferível pois existe um homônimo (embora posterior) para Eugenia herbacea O. Berg, que é E. herbacea A. Chev. publicado em 1908 (Govaerts et al. 2012). Além disso, o nome E. angustissima tem sido mais comumente utilizado nos herbários do Brasil. No Species Link (2012) há 201 coletas identificadas com este nome ao passo que como E. herbacea apenas 18 .

Psidium stritatulum DC., Prodromus 2: 233. 1828. Psidium ramboanum Mattos, nomen novum, Loefgrenia 116: 2. 2001. Psidium nigrum Mattos \& Legrand, Loefgrenia 67: 10. 1975. Nom. illeg., homônimo posterior de $P$. nigrum Lour., Fl. Conchinch. 311. 1790. Nov. Syn. Tipo: Brasil. Mato Grosso, Pantanal, Rio Negro. H.E. Strang 4203 (holotypus, MVM n.v.). 
Quadro 1. Lista das espécies de Myrtaceae com ocorrência comprovada para o estado de Mato Grosso do Sul. Siglas dos herbários de acordo com Thiers (2012) = visto pessoalmente, n.v. = não visto. Determinadores: CEBP = Carolyn Elinore Barnes Proença; DVS = Daniel Villarroel Segarra; JEQF = Jair Eustáquio Quintino de Faria; KMGB = Kadja Milena Gomes-Bezerra; LHSS = Lúcia Helena Soares-Silva; MS = Marcos Sobral; POR = Priscila Oliveira Rosa.

\begin{tabular}{|c|c|c|c|}
\hline Espécie & Coletor & Herbário & Determinador \\
\hline \multirow[t]{2}{*}{$\begin{array}{l}\text { Blepharocalyx salicifolius (Kunth) } \\
\text { O.Berg }\end{array}$} & Conceição, C.A. 1607 & (UB)! & CEBP \\
\hline & Amaral-Júnior, A. 150 & (CGMS, SP, RB, UPCB)! & MS \\
\hline Calyptranthes concinna DC. & Faxina, C. 322 & (CGMS)! & DVS \\
\hline C. lucida Mart. ex DC. & Damasceno-Júnior, G.A. 2616 & (CGMS, HUFU, MBM, UEC, UB)! & CEBP, DVS, KMGB, LHSS \\
\hline $\begin{array}{l}\text { Campomanesia adamantium } \\
\text { (Cambess.) O.Berg }\end{array}$ & Resende, U.B. 142 & (CGMS, RB)! & LHSS \\
\hline C. aurea O.Berg & Pott, V.J. \& Pott, A. 7151 & (CGMS, RB)! & MS \\
\hline C. guazumifolia (Cambess.) O.Berg & Faxina, C. 159 & (CGMS)! & DVS \\
\hline C. pubescens (Mart. ex DC.) O.Berg & Santos, J.F. 4 & (UB)! & CEBP \\
\hline \multirow[t]{2}{*}{ C. sessiliflora (O.Berg) Mattos } & Gomes-Júnior, J.C. 1439 & (SP, UB)! & CEBP \\
\hline & Damasceno-Júnior, G. 4470 & (CGMS)! & MS \\
\hline \multirow[t]{2}{*}{ C. xanthocarpa (Mart.) O.Berg } & Pott, A. 6498 & (CPAP, UB)! & CEBP \\
\hline & Gomes, S.M. 237 & (E, INPA, UB)! & CEBP \\
\hline \multirow[t]{3}{*}{ Eugenia angustissima O.Berg } & Riedel, L. s.n. & (LE n.v., OXF)! & CEBP \\
\hline & Gomes-Júnior, J.C. 2395 & (MBM, SP, UB)! & LHSS, JEQF \\
\hline & Sartori, A. 772 & (CGMS, UB)! & LHSS \\
\hline E. anomala D.Legrand & Hatschbach, G. 76220 & (UB)! & LHSS, JEQF \\
\hline \multirow[t]{2}{*}{ E. aurata O.Berg } & Valio, I.M. 56 & (ESA, NY, SP, UB)! & LHSS, JEQF \\
\hline & Francener, A. 948 & (CGMS, UB)! & LHSS, JEQF \\
\hline E. biflora (L.) DC. & Pott, A. 6510 & (UB)! & LHSS, JEQF \\
\hline E. bimarginata $\mathrm{DC}$. & Simon, M.F. et al. 38 & (UB)! & LHSS, JEQF \\
\hline \multirow[t]{2}{*}{ E. chiquitensis O.Berg } & Oliveira-Filho, E.C. 28 & $(\mathrm{SPF})$ & \\
\hline & Nicásio \& Ochoa s.n. & (CGMS)! & DVS \\
\hline \multirow[t]{2}{*}{ E. dysenterica DC. } & Bridgewater, S. 508A & $(\mathrm{UB}) !$ & LHSS, JEQF \\
\hline & Hatschbach, G. 60923 & $\begin{array}{l}\text { (ESA, INPA, NY, SPSF, MBM, } \\
\text { UPCB)! }\end{array}$ & LHSS \\
\hline \multirow[t]{2}{*}{ E. egensis DC. } & Pott, A. et al. 6566 & (UB)! & LHSS, JEQF \\
\hline & Damasceno-Júnior, G. 2617 & (CGMS, HUFU, UEC, UB)! & LHSS, JEQF \\
\hline \multirow[t]{2}{*}{ E. florida DC. } & Hatschbach, G. 76130 & (MBM, MO, UB, UPCB)! & LHSS, JEQF \\
\hline & Damasceno-Júnior, G. 1638 & (CGMS, HUFU)! & LHSS \\
\hline E. gemmiflora O.Berg & Conceição, C.A. 1922 & (CGMS)! & CEBP \\
\hline E. gracillima Kiaersk. & Mendes, S.W. s.n. & $(\mathrm{RB}) !$ & MS \\
\hline E. hatschbachii Mazine & Pott, A. 10160 & (CGMS)! & MS \\
\hline E. hiemalis Cambess. & Hatschbach, G. 76035 & (FUEL, UB)! & LHSS, JEQF \\
\hline E. involucrata DC. & Faxina, C. 200 & (CGMS)! & DVS \\
\hline E. klotzschiana O.Berg & Hatschbach, G. 51535 & (MBM, MO, SP, UPCB)! & MS \\
\hline E. lambertiana DC. & Conceição, C.A. 1353 & (CGMS, COR, UB)! & LHSS, JEQF \\
\hline \multirow[t]{2}{*}{ E. langsdorffii O.Berg } & Riedel, L. s.n. & (LE) Tipo & \\
\hline & Hatschbach, G. 24653 & (UPCB)! & LHSS, JEQF \\
\hline E. matogrossensis Sobral & Hatschbach, G. et al. 62642 & (BHCB, MBM)! Tipo & MS \\
\hline E. modesta DC. & Barbosa-Filho, W.G. et al. 78 & $(\mathrm{CGMS}) !$ & MS \\
\hline $\begin{array}{l}\text { E. moschata (Aubl.) Nied. ex T.Durand } \\
\text { \& B.D.Jacks. }\end{array}$ & Kuhlmann, J.G. 1064 & $(\mathrm{RB}) !$ & MS \\
\hline E. myrcianthes Nied. & Resende, U.M. 2635 & (CGMS)! & DVS \\
\hline E. neomattogrossensis Mazine & Conceição, C.A. 1716 & (CGMS, UB)! & LHSS, JEQF \\
\hline \multirow[t]{2}{*}{ E. paracatuana O.Berg } & Constantino, R. 119 & $(\mathrm{SP}, \mathrm{UB}) !$ & LHSS, JEQF \\
\hline & Batista-Maria, V.R. 235 & (CGMS) & F. Mazine \\
\hline E. polystachya Rich. & Pott, V.J. 4770 & $(\mathrm{CGMS}) !$ & MS \\
\hline E. pitanga (O.Berg) Nied. & Hatschbach, G. 77199 & (MBM, UB)! & LHSS, JEQF \\
\hline E. punicifolia (Kunth) DC. & Hatschbach, G. 76321 & (INPA, MBM, SPSF, UB)! & LHSS, JEQF \\
\hline E. pyriformis Cambess. & Paula, J.E. de 1859 & $(\mathrm{UB}) !$ & LHSS, JEQF \\
\hline E. repanda O.Berg & Damasceno-Júnior, G.A. 2003 & (UB)! & LHSS, JEQF \\
\hline E. sparsa S.Moore & Damasceno-Júnior, G.A. 2753 & (COR, UB)! & LHSS, JEQF \\
\hline \multirow[t]{2}{*}{ E. stictopetala DC. } & Santos, G.B. 27 & (UB)! & LHSS, JEQF \\
\hline & Pott, A. 4556 & $(\mathrm{COR}, \mathrm{MBM}) !$ & MS \\
\hline
\end{tabular}




\begin{tabular}{|c|c|c|c|}
\hline Espécie & Coletor & Herbário & Determinador \\
\hline E. suberosa Cambess. & Hatschbach, G. 76912 & (MBM, UB)! & LHSS, JEQF \\
\hline E. subterminalis DC. & Hatschbach, G. 77221 & (MBM, UB)! & LHSS, JEQF \\
\hline E. ternatifolia Cambess. & Proença, C.E.B. et al. 1544 & (UB)! & LHSS, JEQF \\
\hline E. uniflora $\mathrm{L}$. & Assis, M.A. 149 & (MBM)! & LHSS \\
\hline Myrcia amazonica DC. & Seramin, A.D. 158 & $(\mathrm{RB})$ & G.M. Barroso \\
\hline M. anomala Cambess. & Ratter, J.A. 5971 & $(\mathrm{E}, \mathrm{K}, \mathrm{MBM}, \mathrm{NY}) !$ & POR \\
\hline M. bella Cambess. & Bridgewater, S. 502 & $(\mathrm{E}, \mathrm{K}, \mathrm{UB}) !$ & POR \\
\hline \multirow[t]{2}{*}{ M. camapuanensis N.Silveira } & Riedel, L. s.n. & $\begin{array}{l}\text { (G, K, LE)! Tipo Aulomyrcia } \\
\text { capitata O. Berg }\end{array}$ & POR \\
\hline & Pott, A. 6496 & (CPAP, UB)! & KMGB, DVS \\
\hline M. citrifolia (Aubl.) Urb. & Dubs, B. 1321 & $(\mathrm{MBM})$ & M.L. Kawasaki \\
\hline M. deflexa (Poir.) DC. & Prance, G.T. 59403 & $(\mathrm{~F}, \mathrm{NY})$ & R. McVaugh \\
\hline \multirow[t]{2}{*}{$\begin{array}{l}\text { M. dictyophylla (O.Berg) Mattos \& } \\
\text { D.Legrand }\end{array}$} & Riedel, L. s.n. & (LE n.v.) Tipo & O.Berg \\
\hline & Assunção, V.A. 443 & (CGMS)! & MS \\
\hline M. goyazensis Cambess. & Hatschbach, G. 51582 & $(\mathrm{MBM}) !$ & MS \\
\hline M. guianensis (Aubl.) DC. & Oliveira, P.I. 31 & (MBM, UB)! & KMGB, DVS \\
\hline M. laruotteana Cambess. & Simon, M.F. et al. 18 & (UB)! & KMGB, DVS \\
\hline \multirow[t]{2}{*}{ M. mollis (Kunth) DC. } & Damasceno-Júnior, G.A. 985 & (CGMS) & M.L. Kawasaki \\
\hline & Resende, V.M. 1417 & (CGMS)! & DVS \\
\hline M. multiflora (Lam.) DC. & Gomes-Júnior, J.C. 2136 & (UB)! & KMGB, DVS \\
\hline M. pinifolia Cambess. & Pott, A. \& Pott, V.J. 8190 & (CGMS, CPAP)! & POR \& CEBP \\
\hline M. racemulosa DC. & Conceição, C.A. 1952 & (CGMS, MBM)! & MS \\
\hline \multirow[t]{2}{*}{ M. myrtillifolia DC. } & Conceição, C.A. 2523 & (CGMS) & \\
\hline & Resende, U.M. 21 & $(\mathrm{RB})$ & G.M. Barroso \\
\hline \multirow[t]{2}{*}{ M. selloi (Spreng.) N.Silveira } & Seleme, E.P. s.n. & (UB)! & KMGB, DVS \\
\hline & Damasceno-Júnior, G.A. 4762 & (CGMS)! & MS \\
\hline M. splendens (Sw.) DC. & Conceição, C.A. 1577 & (CGMS, RB, UB)! & KMGB, DVS \\
\hline M. tomentosa (Aubl.) DC. & Pott, A. 6576 & (CPAP, UB)! & KMGB, DVS \\
\hline M. uberavensis O.Berg & Hatschbach, G. 66570 & (BR, MBM, NY, SP)! & MS \\
\hline M. variabilis DC. & Amaral-Júnior, A. 23 & (CGMS, RB, SP) & M.L. Kawasaki \\
\hline M. vestita DC. & Gomes-Júnior, J.C. 2407 & (UB)! & KMGB, DVS \\
\hline $\begin{array}{l}\text { Myrcianthes pungens (O.Berg) } \\
\text { D.Legrand }\end{array}$ & Damasceno-Júnior, G.A. 1655 & (CGMS, HUFU, MBM)! & MS \& M.L. Kawasaki \\
\hline Myrciaria cuspidata O.Berg & Damasceno-Júnior, G.A. 1895 & (COR, UB)! & DVS \\
\hline M. delicatula (DC.) O.Berg & Hatschbach, G. 46179 & (MBM, MO)! & DVS \\
\hline $\begin{array}{l}\text { M. floribunda (H.West ex Willd.) } \\
\text { O.Berg }\end{array}$ & Damasceno-Júnior, G.A. 2970 & (COR, UB)! & DVS \\
\hline M. rojasii D.Legrand & Hatschbach, G. 58872 & $(\mathrm{MBM}) !$ & MS \\
\hline $\begin{array}{l}\text { Pimenta pseudocaryophyllus (Gomes) } \\
\text { Landrum }\end{array}$ & Bridgewater, S. 508 & (E, UB)! & CEBP \\
\hline Plinia peruviana (Poir.) Govaerts & Damasceno-Júnior, G.A. 2798 & (CGMS)! & CEBP \\
\hline P. rivularis (Cambess.) Rotman & Hatschbach, G. 48488 & $(\mathrm{MBM}) !$ & MS \\
\hline Psidium acutangulum Mart. ex DC. & Damasceno-Júnior, G.A. 2391 & $(\mathrm{COR}) !$ & CEBP \\
\hline \multirow[t]{2}{*}{$\begin{array}{l}\text { P. australe Cambess. var. argenteum } \\
\text { (O.Berg) Landrum }\end{array}$} & Amaral-Júnior, A. 10 & (CGMS, FUEL, SP) & M.L. Kawasaki \\
\hline & Gomes-Júnior, J.C. 1442 & (SP, UB)! & CEBP \\
\hline $\begin{array}{l}\text { P. australe Cambess. var. suffruticosum } \\
\text { (O.Berg) Landrum }\end{array}$ & Rezende, U.M. 1101 & (CGMS, RB)! & CEBP \\
\hline P. grandifolium Mart. ex DC. & Conceição, C.A. 1589 & (CGMS, UB)! & CEBP \\
\hline P. guineense Sw. & Damasceno-Júnior, G. 2917 & (CGMS, HUFU, UB, UEC)! & CEBP \\
\hline P. kennedyanum Morong & Gomes-Júnior, J.C. 1809 & (MBM, SP, UB)! & CEBP \\
\hline \multirow[t]{2}{*}{ P. laruotteanum Cambess. } & Proença, C.E.B. 1550 & (UB)! & CEBP \\
\hline & Amaral-Júnior, A. 178 & (CGMS, SP) & M.L. Kawasaki \\
\hline Psidium myrsinites DC. & Ratter, J.A. et al. 7555 & (UB)! & CEBP \\
\hline Psidium sartorianum (O.Berg) Nied. & Tião \& Ademir s.n. & (HUEM 7975)! & MS \\
\hline Psidium striatulum DC. & Damasceno-Júnior, G. 2658 & (CGMS, UB)! & CEBP \\
\hline
\end{tabular}


A combinação de folhas pequenas, glabras, de ápice e base obtusa, com a face ventral das folhas com venação impressa e glândulas opacas, bem como os frutos solitários, rugosos, costulados, não deixa dúvidas de que se trata de um nome supérfluo para esta espécie de distribuição predominantemente amazônica, mas alcançando o Pantanal.

\section{AGRADECIMENTOS}

Os autores agradecem aos coordenadores pelo convite e à Dra. Ângela Sartori pela sua atenção em visita ao Herbário da Fundação Universidade Federal de Mato Grosso do Sul. Ao Conselho Nacional de Desenvolvimento Científico e Tecnológico/PROTAX, que financiou as bolsas de doutorado de Jair E.Q. de Faria e Priscila O. Rosa e diárias ao D. Villarroel para visitar o Herbário CGMS.

\section{REFERÊNCIAS}

Barbosa-Rodrigues, J. 1903. Myrtaceés du Paraguay recueillies. Imprimerie Typo-lithographique J. Goffin Fils, J. de Grève, Succr. $20 \mathrm{p}$.

Baptista-Maria, V.R., Ribeiro, R.R., Damasceno-Júnior, G.A., Maria, F.S. \& Souza, V.C. 2009. Composição florística de florestas estacionais ribeirinhas no Estado de Mato Grosso do Sul, Brasil. Acta Botanica Brasilica 23(2):535-548.

Battilani, J.L., Ferreira, C.M.M., Penatti, N.C., Vidotto, C., DamascenoJúnior., G.A. \& Pott, A. 2011. Análise comparativa da estrutura de capões de floresta estacional semidecidual na sub-região do Pantanal de Miranda, MS, Brasil, com diferentes métodos de amostragem. In Fitossociologia no Brasil: Métodos e Estudos de Caso (J.M. Felfili, P.V. Eisenlohr, M.M.R.F. Melo, L.A Andrade \& J.A.A. Meira Neto, orgs.). Editora Universidadea Federal de Viçosa, Viçosa, v. 1, p. 520-536.

Berg, O. 1856. Revisio Myrtacearum. Linnaea 27:1-472. 1857. Myrtaceae. In Flora Brasiliensis (C.F.P. Martius \& A.G. Eichler, eds.). F. Fleischer, Lipsiae, v. 14, part. 1, p. 1-468. 1859. Supplementum Myrtacearum. In Flora Brasiliensis (C.F.P. Martius \& A.G. Eichler, eds.). F. Fleischer, Lipsiae, v. 14, part. 1, p. 529-656.

Bueno, N.R., Castilho, R.O., Costa, R.B., Pott, A., Pott, V.J., Sheidt, G.N. \& Batista, M.S. 2005. Medicinal plants used by the Kaiowá and Guarani indigenous populations in the Caarapó Reserve, Mato Grosso do Sul, Brazil. Acta Botanica Brasilica 19(1):39-44.

Bueno, B., Galletti, V.S., Vicentin, W., Damasceno-Júnior, G.A. \& Pott, A. 2011. Comparação de dois métodos fitossociológicos aplicados à mata ciliar do Rio Miranda, Corumbá, MS, Brasil. In Fitossociologia no Brasil: Métodos e Estudos de Caso (J.M. Felfili, P.V. Eisenlohr, M.M.R.F. Melo, L.A Andrade \& J.A.A. Meira Neto, orgs.). Editora Universidade Federal de Viçosa, Viçosa, v. 1, p. 537-556.

Dubs, B. 1998. Prodromus Florae Matogrossensis. Part I. Checklist of Angiosperms. Part II. Types from Mato Grosso. The Botany of Mato Grosso. Series B, n 3. Betrona-Verlag, Küsnacht, p. 444.

Faria, J.E.Q. 2010. O gênero Eugenia L. (Myrtaceae) nos estados de
Goiás e Tocantins, Brasil. Dissertação 250 p. Pós-graduação em Botânica, Universidade de Brasília, Brasília.

Florescer. 2012. Flora Integrada da Região Centro-Oeste. Disponível em: http://florescer.unb.br. Acessado em 25.10. 2012.

Govaerts, R., Sobral, M., Ashton, P., Barrie, F., Holst, B.K., Landrum, L.R., Matsumoto, K., Mazine, F.F., Nic Lughadha, E., Proença, C., Soares-Silva, L.H., Wilson, P.G. \& Luca, E. 2012. World Checklist of Myrtaceae. Facilitated by the Royal Botanic Gardens, Kew. Disponível em: http://apps.kew.org/wcsp/. Acessado em 27.10.2012.

Lucas, E.J., Matsumoto, K., Harris, S.A., Nic Lughadha, E.M., Bernardini, B., \& Chase, M.W. 2011. Phylogenetics, Morphology, and evolution of the large genus Myrcia s.l. (Myrtaceae). International Journal of Plant Sciences 172(7):915-934.

Mamani, F., Pozo, P., Soto, D., Villarroel, D. \& Wood, J.R.I. 2011. Guía Darwin de las plantas de los cerrados de la Chiquitania. Museo de Historia Natural Noel Kempff - Darwin Initiative, Santa Cruz. 212 p.

Mazine, F.F. 2012. Eugenia neomattogrossensis Mazine, a replacement name for Eugenia mattogrossensis Mazine (Myrtaceae). Phytotaxa 69:6.

Pott, A., Pott, V.J., Sciamarelli, A., Sartori, A.L.B., Resende, U., ScreminDias, E., Jacques, E.L., Aragaki, S., Nakajima, J.N., Romero, R.; Cristaldo, A.C.M. \& Damasceno-Júnior., G.A. 2006. Inventário das Angiospermas no Complexo Aporé-Sucuriú. In Biodiversidade do Complexo Aporé-Sucuriú: subsídios à conservação e ao manejo do Cerrado: área prioritária 316-Jauru (T.C.S. Pagotto \& P.R. Souza, orgs.). Editora Universidade Federal do Mato Grosso do Sul, Campo Grande. 308 p.

Proença, C.E.B. 1994. Listagem comprovada das Myrtaceae do Jardim Botânico de Brasília. Boletim do Herbário Ezequias Paulo Heringer 1(1):9-26.

Ratter, J.A., Pott, A., Pott, V.J., Cunha, C.N. \& Haridassan, M. 1988. Observations on woody vegetation types in the Pantanal and around Corumbá. Notes from the Royal Botanic Garden Edinburgh 45:503525.

Romagnolo, M.B. \& Souza, M.C. 2000. Análise Florística e Estrutural de Florestas Ripárias do Alto Rio Paraná, Taquaruçu, MS. Acta Botanica Brasilica 14(2):163-174.

Romagnolo, M.B. \& Souza, M.C. 2004. Os gêneros Calycorectes O.Berg, Hexaclamys O.Berg, Myrcianthes O.Berg, Myrciaria O.Berg e Plinia L. (Myrtaceae) na planície alagável do alto rio Paraná, Brasil. Acta Botanica Brasilica 18(3):613-627.

Silva, D.G. B. da (org.). Egg, M.L.N. (trad.). 1997. Os diários de Langsdorff. Campinas: Associação Internacional de Estudos Langsdorff, Campinas. Editora Fiocruz, Rio de Janeiro. Vol. 2. 400 p.

Silva, J.S.V., Pott, A., Cardoso, E., Moraes, A.S., Salis, S.M., Pott, V.J., Mauro, R.A. \& Galdino, S. 2000. Avaliação Integrada do Maciço do Urucum e Adjacências - Procedimentos e Diretrizes. In Zoneamento Ambiental da Borda Oeste do Pantanal: Maciço do Urucum e Adjacências (J.S.V. Silva org.). Brasília: Embrapa, p. 9-22.

Sobral, M. 2008. Uma nova espécie e duas novas combinações em Myrtaceae do Brasil. Lundiana 9(2):107-110.

Sobral, M., Proença, C., Souza, M., Mazine, F. \& Lucas, E. 2012. Myrtaceae. In Lista de Espécies Flora do Brasil. Jardim Botânico do Rio de Janeiro. Disponível em: http://floradobrasil.jbrj.gov.br/2012/. Acessado em 25.10.2012.

SpeciesLink. 2012. Centro de Referência de Informação Ambiental. Disponível em: www.splink.cria.org.br. Acessado em 28.10.2012.

Thiers, B. Index Herbariorum: A global directory of public herbaria and associated staff. New York. Acessado em 28.10.2012. 